\title{
Learning Performance of Normal and Mutant Drosophila after Repeated Conditioning Trials with Discrete Stimuli
}

\author{
C. D. O. Beck, ${ }^{1}$ Bradley Schroeder, ${ }^{1}$ and Ronald L. Davis ${ }^{1,2}$ \\ Departments of ${ }^{1}$ Molecular and Cellular Biology and ${ }^{2}$ Psychiatry and Behavioral Sciences, Baylor College of Medicine, \\ Houston, Texas 77030
}

A new olfactory conditioning procedure is described using short training trials with discrete presentation of conditioned stimuli (CS) and unconditioned stimuli (US). A short odor presentation along with a single-shock stimulus produced modest but reliable and reproducible learning. Multiple trials presented sequentially improved performance with increasing trial number. Trial spacing had a significant impact on performance. Two trials presented with a short intertrial interval (ITI) produced no improvement over a single trial; two trials with a 15 min ITI significantly boosted performance. This effect required two as- sociative trials, because substituting one of the trials with the CS alone, US alone, or an unpaired CS-US failed to boost performance. The increase in initial performance with two trials decayed within 15 min after training. Thus, the effect is shortlived. The utility of using a battery of tests, including a single short trial, two massed trials, and two spaced trials, to investigate parameters of memory formation in several mutants was demonstrated.

Key words: Drosophila; olfactory conditioning; massed training; spaced training; learning mutant; acquisition
Some of the variables and factors affecting learning after classical conditioning have been elucidated from behavioral studies. The temporal relationship between the presentation of the conditioned stimulus (CS) and the unconditioned stimulus (US) is one important determinant of the probability of forming associations (Rescorla, 1988). Many animals can learn with short delays between the CS and US presence (Rescorla, 1988), but associability with extended delays requires that the subject be aware of the delays (Clark and Squire, 1998). Attention to the appropriate cues is also of profound importance in memory formation because of a limitation for processing information (Miller, 1999). A final example of an important variable is trial spacing. Trial spacing effects have a pronounced effect on the durability of memory formed, with task performance, in general, being elevated with trial spacing. This has been shown to be true for taste aversion, fear, and eyeblink conditioning (Yin et al., 1994; Carew, 1996).

Drosophila have been used to study the genetics and molecular biology of learning (Davis, 1996). Numerous learning assays have been devised for evaluating overall performance based on the presentation of different types of cues (Davis, 1996). One robust task is differential odor conditioning in which flies are tested for their ability to associate an odor CS + with a US of electric shock in opposition to a second odor CS- without shock (Jellies, 1981; Tully and Quinn, 1985; Davis, 1996). Flies will learn to alter the orientation of their flight after operant or classical conditioning, when visual cues are paired with heat as a reinforcer or US (Wolf et al., 1998). Moreover, a male fly's courtship intensity of virgin

\footnotetext{
Received Dec. 2, 1999; revised Jan. 27, 1999; accepted Jan. 27, 2000.

This research was supported by National Institutes of Health Grant NS19904, the Mathers' Charitable Foundation, and the R. P. Doherty-Welch Chair in Science to R.L.D. We thank Gregg Roman, Yuzhong Cheng, and Dirk Jones for critical comments on this manuscript.

Correspondence should be addressed to Ronald L. Davis, Department of Molecular and Cellular Biology, Baylor College of Medicine, One Baylor Plaza, Houston, TX 77030. E-mail: rdavis@bcm.tmc.edu.

Copyright (C) 2000 Society for Neuroscience $0270-6474 / 00 / 202944-10 \$ 15.00 / 0$
}

females is subject to conditioned suppression by previous courtship bouts with mature females (Griffith et al., 1993).

These and other Drosophila behavioral assays (Davis, 1996) have been used to evaluate the learning performance of several different mutants. The earliest isolated mutants included dunce (Dudai et al., 1976) and rutabaga (Livingstone et al., 1984). These mutants were subsequently shown to have lesions in genes that encode components of the cAMP signaling pathway (Byers et al., 1981; Davis and Kiger, 1981; Chen et al., 1986; Levin et al., 1992). In addition, the gene products were found to be preferentially expressed within the mushroom bodies (Nighorn et al., 1991; Han et al., 1992). These observations, along with other results suggesting the importance of mushroom bodies (Davis, 1993, 1996), provided a rationale for modeling the effects of cAMP signaling on mushroom body cell physiology to influence organismal behavior (Davis, 1993, 1996). The themes of cAMP signaling and mushroom body importance have continued in recent years (Skoulakis et al., 1993; DeBelle and Heisenberg, 1994; Yin et al., 1994), although the characterization of additional mutants has provided other players that may perform roles independently of the cAMP pathway (Skoulakis and Davis, 1996; Grotewiel et al., 1998). Nevertheless, remarkably little is known about the specific ways in which the identified molecules affect overall learning or memory. This is, in part, because of the lack of appropriate behavioral assays designed to extract deeper insights, including for example, whether any given mutant affects the optimal timing [intertrial interval (ITI)] of multiply presented cues or the rate of acquisition.

In the work presented here, we focused on early memory processing, the critical period immediately after training. We developed learning procedures based on the use of a short training trial with discretely presented stimuli. This paradigm permitted the dissection of the behavioral processes that occur shortly after training and before long-term memories form. A short discrete conditioning trial offers several advantages. First, in a short trial involving a single shock US rather than the multiple 
shocks often used, the association between the CS and the US is less ambiguous, and therefore results from such a procedure are easier to interpret. Second, a short discrete trial produces less than maximal learning, thus allowing examination of the effects of cumulative training on early memory. Third, the modest learning produced by the short trial procedure alleviates concerns about hitting ceiling levels of performance. Fourth, the use of short, multiple training trials allowed us to investigate the effects of different intertrial intervals on performance. As a result, the short trials with discrete stimuli offered a novel way to evaluate the effects of learning mutants on memory processes.

\section{MATERIALS AND METHODS}

Fly stocks and culture. Five fly stocks were used in this study. The control in all cases was a Cantonized rosy $\left(r y^{506}\right)$ stock that had an isogenic third chromosome carrying the ry mutation (Han et al., 1992). This stock is the progenitor to the mutants in this study and has been used as a control for wild-type behavior in several previous studies (Han et al., 1992; Skoulakis et al., 1993; Skoulakis and Davis, 1996; Grotewiel et al., 1998). The rutabaga allele rut ${ }^{2080}$ was isolated as a enhancer detector element at the rut locus of the $r y^{506}$ control line (Han et al., 1992). The $\mathrm{Vol}^{1}$ mutant contains an enhancer detector element at the Volado locus of the $r y^{506}$ control. $\mathrm{Vol}^{2}$ is a small deletion of the locus, and $\mathrm{Vol}^{2}, h s p \mathrm{Vol}-\mathrm{s}$ has the $\mathrm{Vol}^{2}$ lesion along with a heat-inducible transgene carrying the Voladoshort (Vol-s) cDNA (Grotewiel et al., 1998). The latter stock was previously named VS-T3 (Grotewiel et al., 1998).

Flies were seeded into dry bottles using 20-40 flies per bottle and cultured at $22^{\circ} \mathrm{C}$. They were transferred into another bottle after $7 \mathrm{~d}$, and adult progeny were collected 14-18 d later. This was performed with short $\mathrm{CO}_{2}$ anesthesia, and groups of $\sim 40$ flies were transferred to clean and dry food vials. Collection was performed at least $2 \mathrm{hr}$ before the onset of training. For some experiments, groups of flies were heat shocked at $37^{\circ} \mathrm{C}$ for $15 \mathrm{~min}$ in preheated vials and then transferred to vials held at $22^{\circ} \mathrm{C}$ until training. Flies were fed $35 \mathrm{~mm}$ cycloheximide in a sugar solution for $12 \mathrm{hr}$ for some experiments (Tully et al., 1994).

Olfactory classical conditioning and odor avoidance. Differential conditioning involved the presentation of one of two odors (CS+) with electric shock (US), followed by presentation of the second odor (CS-) with the absence of shock. The two odorants, 3-octanol and benzaldehyde, were used as the odor sources for the CS + and $\mathrm{CS}-$ in a counterbalanced design, with half of the flies used for the calculation of each performance index being trained to octanol and the other half to benzaldehyde. Training was performed under dim red light at $21-24^{\circ} \mathrm{C}$ and at $63-68 \%$ humidity. The electric shock was delivered to the flies through an electrifiable and flexible copper grid that served as the floor of a small Plexiglas training tube.

The procedures used for "long program" training and "short program" training were identical except for schedule. Long program training used $\mathrm{CS}+$ odor presentations of $60 \mathrm{sec}$ accompanied by 12 shock pulses $(1.25$ sec duration) delivered once every $5 \mathrm{sec}$. The final version of short program training used odor presentations of $10 \mathrm{sec}$ accompanied by one shock (1.25 sec duration) delivered $8 \mathrm{sec}$ after odor onset.

Trained flies were tested in a T-maze in which the two odors were simultaneously presented to flies starting in the center of the maze. The flies were permitted to move freely in the maze during the 2 min test. Naive flies exhibited no preference between the two odors. Memory was indicated by approaching the $\mathrm{CS}-$ and avoiding the $\mathrm{CS}+$. After the 2 min test, flies were anesthetized, collected, and counted. A performance index was calculated as the percentage of flies that correctly avoided the $\mathrm{CS}+$ minus the percentage that incorrectly avoided the CS-. Thus, naive flies would show a performance index of zero, whereas flies expressing perfect memory would show a performance index of 100 .

Odor avoidance was as described in the text using the T-maze and odors used for odor conditioning. In this test, however, one odor was replaced by a flow of fresh air. Statistical analyses were performed using ANOVAs with planned comparisons or exhaustive post hoc comparisons as appropriate.

\section{RESULTS}

\section{Short training trials produce modest but stable performance}

The traditional procedure for associative olfactory training (long program) uses 12 electric shock pulses (duration $1.25 \mathrm{sec}$ each) given at $5 \mathrm{sec}$ intervals over a $1 \mathrm{~min} \mathrm{CS}+$ odor exposure (Jellies, 1981; Tully and Quinn, 1985; Grotewiel et al., 1998). This is followed by $30 \mathrm{sec}$ of fresh air, $1 \mathrm{~min}$ CS - odor exposure, and then 30-45 sec of fresh air before trial termination. The duration of the $\mathrm{CS}+$ and $\mathrm{CS}-$ exposure was truncated in different groups, and a proportional number of shocks were presented at the same frequency ( 1 shock every $5 \mathrm{sec}$ ). We observed a decrease in the performance index (Fig. 1a) with a decrease in CS duration and shock number. It is noteworthy that even a single shock presented $8 \mathrm{sec}$ into a $10 \mathrm{sec} \mathrm{CS}+$ exposure was sufficient to reliably produce significant learning.

It was possible, however, that the lower level of performance observed after training with a $10 \mathrm{sec} \mathrm{CS}+$ exposure and a single shock was in part attributable to a failure of some of the flies to perceive the brief stimuli and acquire the association. To test this possibility, flies were trained using a single trial consisting of a 10 sec CS exposure and a single shock and then tested and sequestered (without anesthesia) into two groups according to whether they responded correctly (avoiding the $\mathrm{CS}+$ ) or incorrectly (avoiding the $\mathrm{CS}-$ ). These two groups were immediately retested without further training to examine whether flies that responded correctly on the first test would again avoid the CS+ and whether the flies that responded incorrectly would again fail to avoid the $\mathrm{CS}+$. If the flies that failed to avoid the CS+ in the first test did so because they had not perceived the brief stimuli during training, then one would expect these flies to fail in the retest as well. Conversely, if all flies perceived the stimuli and the performance index simply reflects the probability that any individual responds correctly after forming a weak association between the CS+ and the US, then one would expect the flies in the correct and incorrect groups to show equivalent performance in the retest. This was the case (Fig. 1b). Flies that responded correctly in the first test performed the retest equivalently to flies that responded incorrectly in the first test. This indicated that the performance index reflects the probability of making the correct choice and that an association had formed in most, if not all, trained flies (Tully et al., 1994).

The effect of a longer US during the $10 \mathrm{sec}$ CS exposure was examined by comparing performance with $1.25,5$, and $10 \mathrm{sec}$ shock pulses during a single trial. There was no significant effect of a longer US on performance (Fig. 1c). Trials consisting of a short duration shock $(1.25 \mathrm{sec})$ given $8 \mathrm{sec}$ into the $10 \mathrm{sec} \mathrm{CS}+$ exposure were used in the experiments discussed below. This is subsequently referred to as the short program.

\section{Effects of two trials and intertrial interval}

Studies of many species have shown that when training involves multiple trials, the time interval between trials is an important variable to the efficacy of the accumulated training (Carew et al., 1972; Fanselow and Tighe, 1988; Tully et al., 1994; Spieler and Balota, 1996; Kogan et al., 1997; Hermitte et al., 1999; Muzzio et al., 1999). To determine the optimal ITI for SHORT PROGRAM conditioning, groups of flies were given two trials with different ITIs ranging from $30 \mathrm{sec}$ to $1 \mathrm{hr}$. Between trials, the flies remained in the training tubes. Immediately after training, flies were tested as described above. Gains in performance were referenced to a control group that received only a single trial. 
(a)

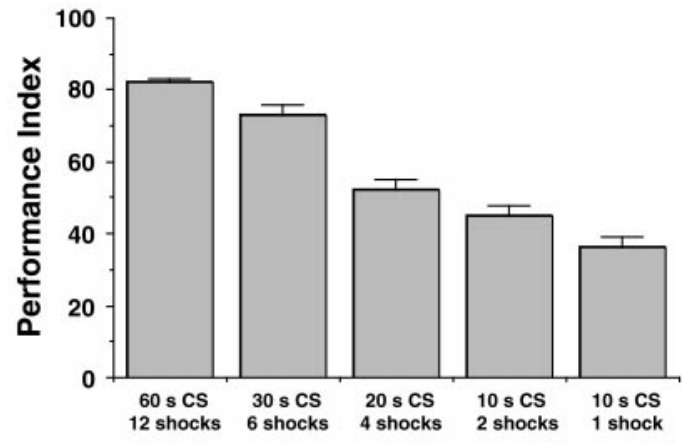

(b)

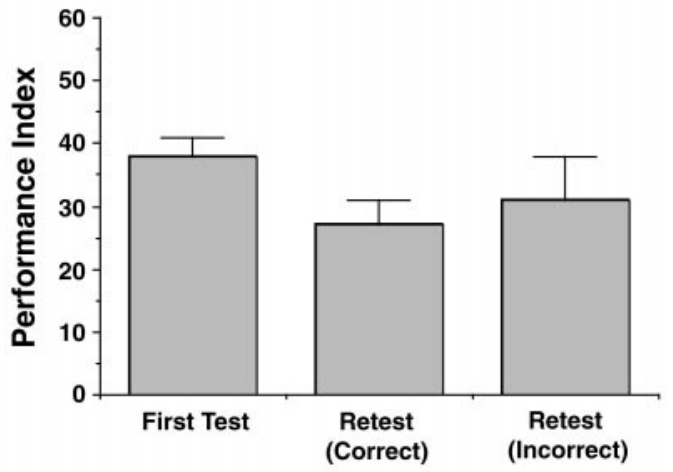

(c)

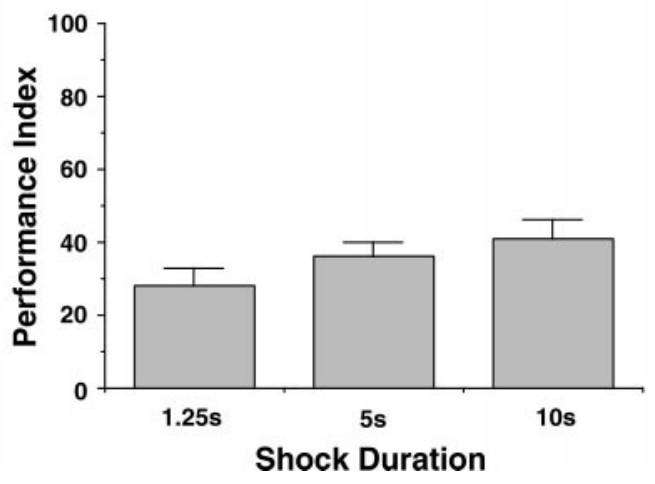

Figure 1. Performance with altered CS and US duration. $a$, Performance decrement with decreased CS exposure and US number. $b$, Performance of flies in a retest that had made a previous correct or incorrect choice. $c$, Effects of increasing electric shock duration with a $10 \mathrm{sec}$ CS + exposure. No significant effect was observed, although there was a trend toward high performance with longer US exposure. Statistics are as follows. $a$, Onefactor ANOVA revealed a significant effect $\left(F_{(4,25)}=51.1 ; p<0.01 ; n=\right.$ 6 in each group) between groups. Fisher post hoc comparisons revealed significant differences between all groups. A two-tailed, one-sample $t$ test with population mean of 0 revealed the $10 \mathrm{sec}, 1$ shock condition produced significant conditioning $\left(t_{(5)}=11.1 ; p<0.01\right)$. $b$, One-factor ANOVA was not significant $\left(F_{(2,24)}=1.07\right.$;S; $n=9$ for all groups $)$. Mean $\pm \mathrm{SEM}$; flies in first test, $38 \pm 3$; correct flies in retest, $27 \pm 4$; incorrect flies in retest, $31 \pm 7$. $c$, One-factor ANOVA revealed no significant effect $\left(F_{(2,15)}=1.9 ; \mathrm{NS} ; n=6\right.$ per group $)$. Mean $\pm \mathrm{SEM} ; 1.25 \mathrm{sec}, 28 \pm 5 ; 5 \mathrm{sec}$, $36 \pm 4 ; 10 \mathrm{sec}, 41 \pm 5$.

The ITI had a significant effect on the performance of the flies in the test (Fig. 2). Intertrial intervals of 10 and 15 min boosted performance over a single trial; the performance score with a 15 min ITI was $160 \%$ of the single-trial value. Performance after training at $30 \mathrm{sec}, 1 \mathrm{~min}$, and $5 \mathrm{~min}$ ITIs were not significantly different from performance after training with a single trial. This indicates that there is an advantage to spacing the trials a minimal interval, perhaps because of the processing mechanisms underly-

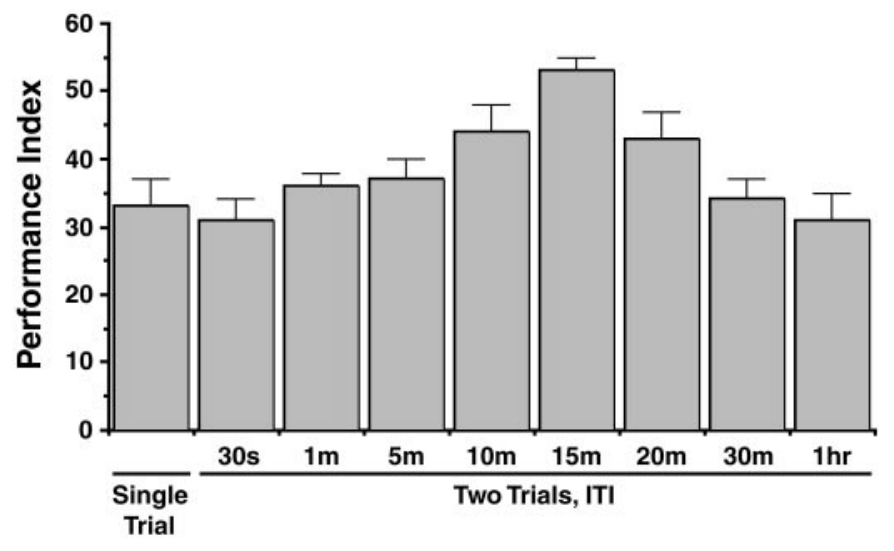

Figure 2. Effect of intertrial interval. Performance was measured after a single trial or after two trials separated by the time indicated. Optimal performance occurred with ITIs of 10 and $15 \mathrm{~min}$. Statistics are as follows: one-factor ANOVA revealed a significant effect of ITI $\left(F_{(8,45)}=\right.$ $4.7 ; p<0.01 ; n=6$ per group). Fisher post hoc comparisons revealed that performance with a 15 min ITI was significantly elevated over all other groups, except after training at a $10 \mathrm{~min}$ ITI.

ing short-term memory for the first trial. It is interesting to note that ITIs longer than 15 min did not confer an advantage; Fisher post hoc comparisons showed that training with $20 \mathrm{~min}, 30 \mathrm{~min}$, or $1 \mathrm{hr}$ ITIs did not result in a performance level significantly greater than that after a single trial. This may be because shortterm memory for the first trial begins to fade after $15 \mathrm{~min}$ and cannot be reinforced by the effects of the second trial. Curiously, the optimum ITI of $15 \mathrm{~min}$ is the same as the optimal interval for obtaining long-term memory using spaced trials of the long program (Tully et al., 1994). For subsequent experiments, we used 30 sec and 15 min ITI training procedures to represent the effects on performance of massed and spaced training, respectively.

\section{Performance after multiple trials at two different ITIs}

Groups of flies received 1-20 trials at either $30 \mathrm{sec}$ or 15 min ITIs. Training at both ITIs produced a higher level of performance with a greater number of trials, but the two ITIs affected performance differently (Fig. 3a). Consistent with the results above, post hoc comparisons showed that training with two trials at a $15 \mathrm{~min}$ ITI produced a significantly higher level of performance than a single trial or two trials at a $30 \mathrm{sec}$ ITI. After three trials at a 30 sec ITI, performance levels were significantly greater than after a single trial, and performance after 10, 15, or 20 trials at a $30 \mathrm{sec}$ ITI was greater than that after three trials. With a 15 min ITI, there was no significant increase in performance between two and five trials. However, after seven trials, there was a significant increase in the level of performance that remained high after 10 trials and then dropped after 15 or 20 trials. For training at a 15 min ITI, the length of training sessions may have been a confounding factor, in that a training session for two trials at a $15 \mathrm{~min}$ ITI required $20 \mathrm{~min}$, whereas a training session for 10 trials at a $15 \mathrm{~min}$ ITI required $2.5 \mathrm{hr}$.

To test the effects of training session length on performance, an additional experiment was performed with three groups, one receiving five trials at a $15 \mathrm{~min}$ ITI, the second receiving 10 trials at a 15 min ITI, and the last receiving five trials at a $30 \mathrm{~min}$ ITI (Fig. $3 b$ ). The five trials, $15 \mathrm{~min}$ ITI group and the five trials, 30 min ITI group obviously received the same number of trials, and the 10 trials, $15 \mathrm{~min}$ ITI group and the five trial, $30 \mathrm{~min}$ ITI group received a training session of the same duration, $2.5 \mathrm{hr}$. If the 


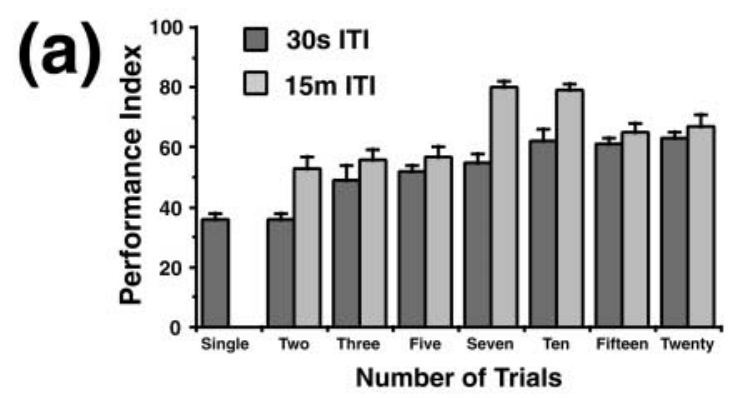

(b)

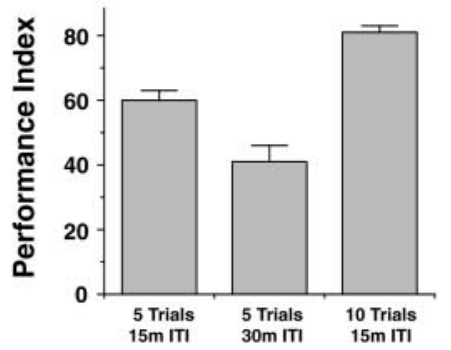

(c)

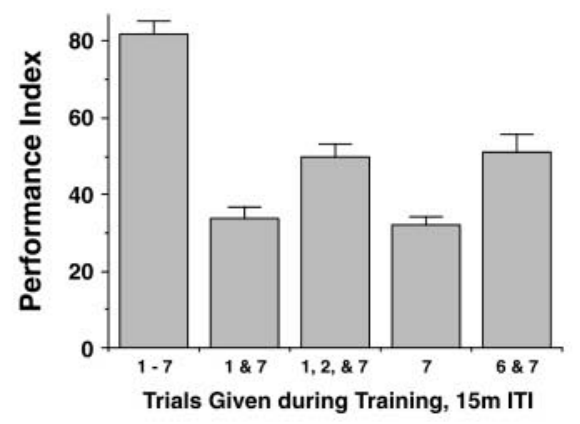

(d)

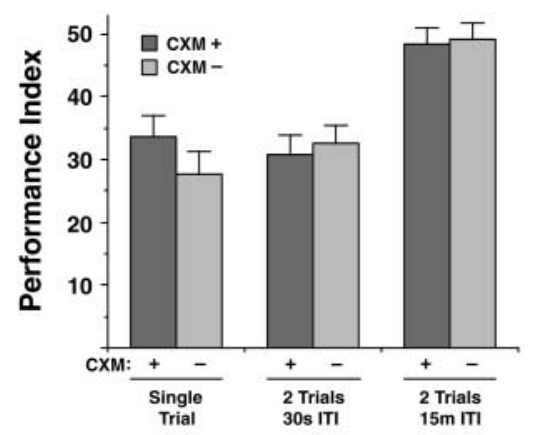

(e)

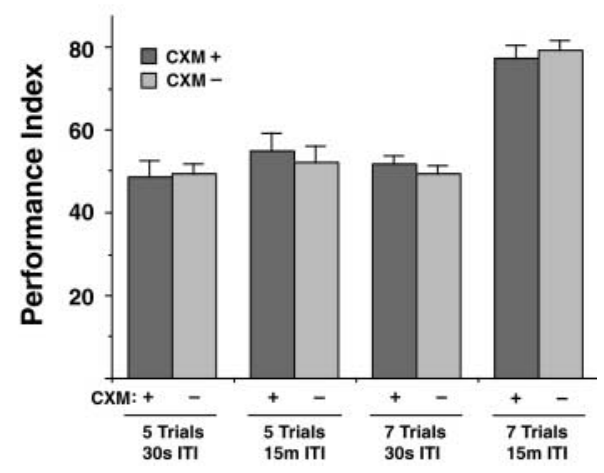

training session length conveyed a significant advantage because of contextual cues or other factors, the five trials, $30 \mathrm{~min}$ ITI group would be expected to perform better than the five trials, 15 min ITI group. If, on the other hand, the number of trials is the important factor in the difference between performance levels after 5 and 10 trials at a $15 \mathrm{~min}$ ITI, the five trials, $30 \mathrm{~min}$ ITI group should not perform better than the five trials, 15 min ITI group. This was the case; the performance after five trials at a 30 min ITI was significantly lower than the performance after five trials at a $15 \mathrm{~min}$ ITI or 10 trials at a $15 \mathrm{~min}$ ITI. Thus, the

\section{$\leftarrow$}

Figure 3. Effect of multiple trials at two different ITIs. $a$, Different groups were trained with 1-20 trials at either a $30 \mathrm{sec}$ or $15 \mathrm{~min}$ ITI. Two trials at a $30 \mathrm{sec}$ ITI did not significantly increase performance over one trial, but subsequent trials at this ITI elevated performance. Two trials at a 15 min ITI, however, produced significantly higher performance than a single trial or two trials at a $30 \mathrm{sec}$ ITI. A further significant jump in performance was observed with groups given seven trials at a 15 min ITI. $b$, Effect of training session length. Three groups were trained, two receiving the same number of training trials, and two receiving the same total training session time. Trial number was the important factor in elevating performance. $c$, Performance after presenting a subset of seven, 15 min spaced training trials. Each group was trained concurrently over the time required for seven spaced trials, but the different groups received only the training trials indicated. Seven-trial training produced robust conditioning. The performance increase observed between five and seven spaced training trials (Fig. 3a) was not reproduced by presenting only trials 1 and $7 ; 1,2$, and 7 ; or 6 and $7 . d, e$, Effects of cycloheximide treatment on flies given two or seven spaced training trials. Flies were fed $35 \mathrm{~mm}$ cyclohexmide in $4 \%$ sucrose for $12 \mathrm{hr}$ before training. Different groups then received single trial, two trials $30 \mathrm{sec}$ ITI, two trials $15 \mathrm{~min}$ ITI, 5 trials $30 \mathrm{sec}$ ITI, 5 trials $15 \mathrm{~min}$ ITI, 7 trials $30 \mathrm{sec}$ ITI, and 7 trials 15 min ITI. The performance enhancement of spaced training with two trials $(d)$ and spaced training with seven trials $(e)$ was reproduced. The enhanced performance was independent of cyclohexmide treatment. Statistics are as follows. $a$, One-factor ANOVA revealed a significant effect of trial number at both ITIs: $30 \mathrm{sec}$ ITI, $F_{(7,45)}=11.0 ; p<0.01 ; n=6-8$ per group; $15 \min$ ITI, $F_{(7,45)}=23.5 ; p<0.01 ; n=6-8$ per group. The ITI affected performance (two-factor ANOVA; effect of ITI, $F_{(1,78)}=41.5$; $p<0.01$; effect of trials, $F_{(6,78)}=16.0 ; p<0.01$; interaction of ITI $\times$ trials, $F_{(6,78)}=3.0 ; p<0.02$. Post hoc comparisons showed significant increases in performance at a $30 \mathrm{sec}$ ITI between two and three trials, and between three and 10 trials. Similar comparisons for groups trained with a 15 min ITI showed significant increases between one and two trials, and between two and seven trials. $b$, One-factor ANOVA showed that all three groups were different $\left(F_{(2,15)}=31.7 ; p<0.01 ; n=6\right.$ per group). Mean \pm SEM; 5 trials 15 min ITI, $60 \pm 3 ; 10$ trials 15 min ITI, $81 \pm 2 ; 5$ trials 30 min ITI, $41 \pm 5$. $c$, There was an overall effect of training condition $\left(F_{(4,29)}=33.1 ; p<0.01\right)$. Fisher post hoc revealed significant differences between all groups and the group receiving all seven trials. Mean \pm SEM; trials $1-7,82.0 \pm 3.2$; trials 1 and $7,33.7 \pm 3.0$; trials 1,2 , and 7, $49.8 \pm$ 3.6 ; trial 7, 32.2 \pm 2.1 ; trials 6 and 7, $51.0 \pm 4.9 ; n=6$ per group. $d$, There was a significant effect of training $\left(F_{(2,30)}=21.3 ; p<0.01 ; n=6\right.$ per training condition-treatment). There was no significant effect of cyclohexmide $(C X M)$ treatment $\left(F_{(1,30)}=0.2 ; \mathrm{NS} ; n=6\right.$ per training condition-treatment) and no significant interaction between training condition and cyclohexmide treatment $\left(F_{(2,30)}=0.9 ; \mathrm{NS} ; n=6\right.$ per training condition-treatment). Fisher post hoc revealed significant differences between two trials, 15 min ITI conditions (with or without cyclohexmide) and both other training conditions. $e$, There was a significant effect of training $\left(F_{(1,40)}=35.6 ; p<0.01 ; n=6\right.$ per training condition-treatment $)$ and $\operatorname{ITI}\left(F_{(1,40)}=53.4 ; p<0.01 ; n=6\right.$ per training condition-treatment $)$, and a significant interaction between training trials and ITI $\left(F_{(1,40)}=\right.$ 27.3; $p<0.01 ; n=6$ per training condition-treatment $)$. There was no significant effect of cyclohexmide treatment $\left(F_{(1,40)}=0.9 ; \mathrm{NS} ; n=6\right.$ per training condition-treatment) nor significant interactions of cyclohexmide treatment with training trials $\left(F_{(1,40)}=0.4 ; \mathrm{NS} ; n=6\right.$ per training condition-treatment $)$ or ITI $\left(F_{(1,40)}=0.1 ; \mathrm{NS} ; n=6\right.$ per training condition-treatment). Fisher post hoc revealed significant differences between seven trials, 15 min ITI conditions (with or without cyclohexmide) and all other groups. 
number of training trials and not training session length was responsible for improved performance with 10 over 5, spaced trials.

Training with multiple, spaced trials revealed a reproducible jump in performance between trials 1 and 2 and between trials 5 and 7 (Fig. 3a). The enhancement that occurred between five and seven trials $(\sim 100$ min total training time) prompted the question of whether all seven training trials were required to produce the effect or whether some combination of early trials along with an appropriate time delay (time until the seventh trial and test) in the training tube was responsible. Different groups of flies were trained with seven spaced training trials or a subset of these (Fig. $3 c$ ). Flies that received the first trial and the seventh performed no better than flies that had received only one training trial (the seventh). Nor did groups that received the spaced effect of two trials (6 and 7) or the spaced effect of two trials along with the seventh trial $(1,2$, and 7$)$ exhibit performance like the group receiving all seven. Therefore, the full effect requires all seven training trials.

It has been frequently observed that the enhancement in performance caused by spaced trials is sensitive to inhibitors of transcription or translation (Montarolo et al., 1986; Tully et al., 1994; Martin et al., 1997; Hermitte et al., 1999). For example, the prolonged memory after spaced, long program training in Drosophila is sensitive to inhibitors of protein synthesis. Furthermore, enduring enhancements in synaptic transmission attributable to spaced application of neuromodulators have been demonstrated to be protein synthesis-dependent.

The enhancement in performance observed after two spaced trials occurred at $20 \mathrm{~min}$ into the training program, a time seemingly too short to be a result of altered protein synthesis. The enhancement noted with seven spaced trials occurred at $105 \mathrm{~min}$ into the training program, a lag potentially long enough to accommodate a change in protein synthesis. To test the possibility that the performance enhancements with two or seven spaced trials were protein synthesis-dependent, groups of flies were fed cyclohexmide using procedures that block the effect of spaced training for long program conditioning (Tully et al., 1994).

The treatment failed to have any effect on the enhancement with two or seven spaced trials (Fig. 3d,e). A 50\% increase in performance index was observed with or without cyclohexmide treatment after two spaced trials compared with two massed trials (Fig. $3 d$ ). Approximately the same performance increase was observed with or without cyclohexmide treatment after seven spaced trials compared with seven massed trials (Fig. 3e). The enhanced performance observed here was therefore independent of protein synthesis and must represent mechanistically a new effect of spaced training.

\section{Factors influencing performance with two-trial, spaced training}

The remarkable increase in performance with two spaced trials over two massed trials (Figs. 2, 3) led us to investigate the training factors that might be responsible. The time of exposure to the training apparatus was different between two massed trials and two spaced trials and was therefore a potential factor influencing the level of performance. To test the effect of apparatus exposure, one group was trained with two trials at a $30 \mathrm{sec}$ ITI at the end of a $17 \mathrm{~min}$ period spent in the training apparatus (Fig. $4 a$ ). In addition, a group was trained with two trials at a $30 \mathrm{sec}$ ITI at the beginning of a $17 \mathrm{~min}$ period spent in the training apparatus (Fig. $4 b$ ). In both cases, control groups receiving a single trial, two
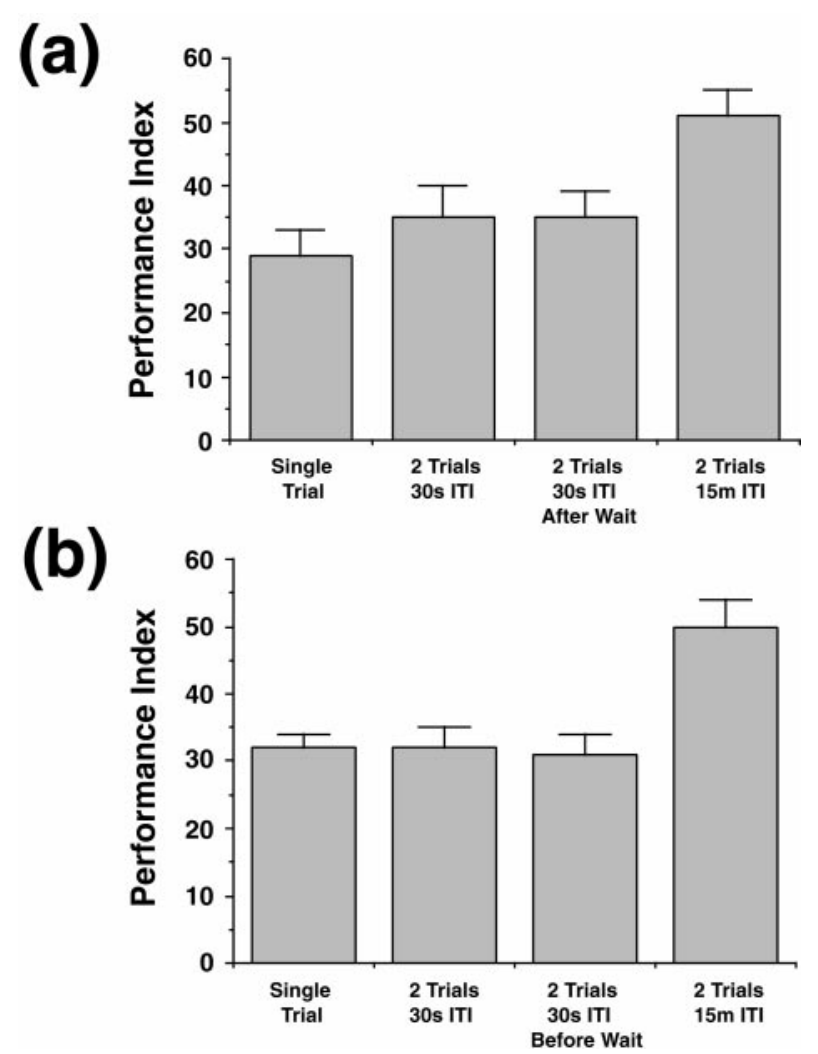

Figure 4. Effects of apparatus exposure on two-trial performance. $a$, An experimental group received two massed training trials after exposure to the apparatus for $17 \mathrm{~min}$ to make the total exposure time equivalent to the two trials, 15 min ITI group. $b$, An experimental group received two massed training trials before an additional $17 \mathrm{~min}$ exposure to the apparatus before the test. Statistics are as follows. $a$, Training after time in apparatus, $F_{(3,20)}=9.1 ; p<0.01 ; n=6$ per group. $b$, Training before time in apparatus, $F_{(3,20)}=4.8 ; p<0.01 ; n=6$ per group. Post hoc comparisons showed that the performance of all groups trained at a $30 \mathrm{sec}$ ITI was significantly less than those trained at a $15 \mathrm{~min}$ ITI and was not different from those groups that received a single trial in training.

massed trials, and two spaced trials were included. Additional time spent in the training apparatus either before or after two massed trials failed to elevate performance to that of groups receiving two spaced trials. Rather, the groups receiving additional exposure to the apparatus performed like groups receiving single trial conditioning. Therefore, additional training apparatus exposure cannot account for the increased performance observed with spaced training.

It was also possible that the advantage conveyed by two trials, spaced training, was attributable to factors other than associative conditioning. In a phenomenon described as perceptual learning, preexposure to a CS before the associative conditioning enhances performance resulting from the training, perhaps by heightening the subject's awareness of the CS before the training begins (Goldstone, 1998). It was possible, therefore, that preexposure to the CS in lieu of an initial associative trial followed by an associative trial after a $15 \mathrm{~min}$ interval would be sufficient to produce the higher level of performance. Conversely, preexposure to the CS may instead produce latent inhibition, a phenomenon in which preexposure to the CS decreases the potential for that stimulus to be associated with a US in subsequent training, perhaps because the subject learns that the CS is irrelevant (Weiner, 1990; Lubow, 1997). Latent inhibition would be exposed 
as a lower level of performance by groups exposed to the CS alone and then trained with a single associative trial after a 15 min wait compared with groups given a single trial with no CS preexposure.

The effects of exposure to the CS alone in the second of the two trials were also examined. In this case, a single associative trial was followed by a $15 \mathrm{~min}$ interval and then presentation of the CS alone. If sensitization to the CS occurs during CS alone presentation, one might expect performance to be enhanced. On the other hand, if the CS alone presentation after an associative trial acts as an extinction trial, performance might be lower than that produced by a single associative trial.

Presentation of the US alone, or the CS and US together but unpaired, may also affect performance. As with the presentation of the CS alone, presentation of the US alone may enhance or disrupt performance produced by one associative trial. Presentation of the CS and US unpaired should not enhance performance because the explicit dissociation of the CS and US should weaken any existing association or inhibit the ability to form an association.

We examined the effects of replacing either the first (Fig. $5 a$ ) or the second (Fig. $5 b$ ) of the two spaced trials with the US alone, the CS alone, the CS and US unpaired (30 sec apart), or "air" (instead of odor carried in air) only. Two control groups that received, respectively, two trials at a $15 \mathrm{~min}$ ITI and a single trial only were included in each experiment. As shown in Figure $5 a$, there was an overall effect of training; post hoc comparisons showed that the performance of all groups was significantly lower than that of the group receiving two trials at a 15 min ITI (CS-US paired). The performance of the groups receiving air only, US alone, and CS alone as a first trial was not different from the single-trial group. Thus, no evidence for effects of the CS or US alone was obtained. However, the performance of the group receiving the CS and US unpaired as a first trial was significantly lower than the single-trial group. Thus, the dissociation of the CS and the US in the unpaired trial weakened the formation of the association in the paired trial 15 min later.

The results of replacing the second of two trials are shown in Figure $5 b$. The performance of all groups was significantly lower than that of the group receiving training with two paired trials at a 15 min ITI (CS-US paired). The performance of the groups receiving air only, US alone, and CS alone was not different from the group receiving a single trial. However, as before, the group receiving CS-US unpaired in the second trial showed a lower level of performance than the single-trial group. Thus, dissociation of the CS and US, 15 min after the associative trial, weakened the CS-US association produced by single-trial training.

Together, the results from these two experiments show that the advantage conveyed by training with two trials at a 15 min ITI requires the presentation of two full associative trials. Presentation of the CS alone or the US alone during the first or second trial is insufficient and results in performance similar to that after a single trial. When the CS and US are explicitly dissociated during the first or second trial, performance decreases relative to that after a single trial.

\section{Memory after two-trial, spaced training}

The pronounced effect that two spaced trials has on immediate performance prompted us to examine the effects that this training has on later memory. Retention of performance was measured after training with a single trial, two trials with a $30 \mathrm{sec}$ ITI, and two trials with a 15 min ITI. Measurements were made at $3 \mathrm{~min}$
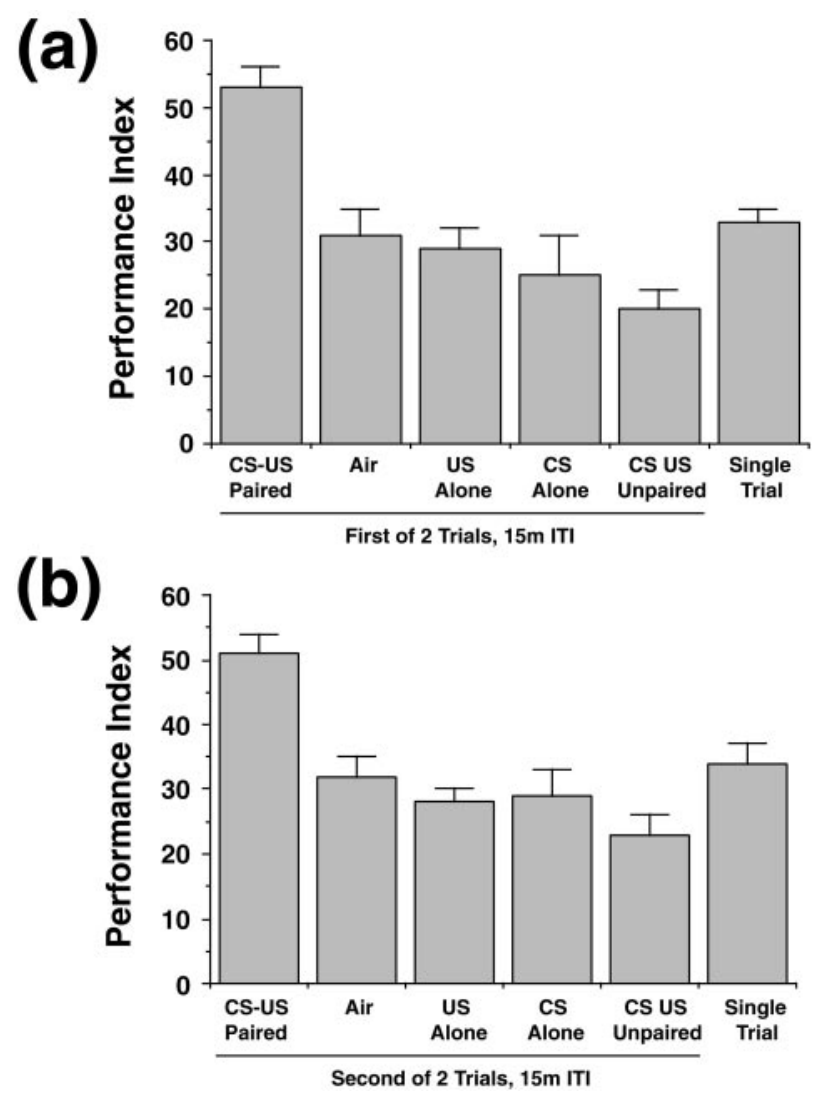

Figure 5. Effects of air, US alone, CS alone, or CS-US unpaired on two-trial spaced training. $a$, Two-trial conditioning with a 15 min ITI produced performance significantly elevated over groups conditioned with a single trial. Substitution of the first $(a)$ or second (b) CS-US pairing with air, US alone, or CS alone produced performance equivalent to a single conditioning trial. Unpairing the CS and US on the first $(a)$ or second $(b)$ trial inhibited the association. Statistics are as follows. $a$, There was an overall effect of training $\left(F_{(5,30)}=9.1 ; p<0.01 ; n=6\right.$ per group). Fisher post hoc revealed no differences between the single-trial group and the groups given air, US alone, and CS alone. Significant differences were observed between the single-trial group and the CS-US paired and CS-US unpaired groups. $b$, There was an overall effect of training $\left(F_{(5,30)}=10.2 ; p<0.01 ; n=6\right.$ per group). Fisher post hoc revealed no differences between the single-trial group and the groups given air, US alone, and CS alone. Significant differences were observed between the single-trial group and the CS-US paired and CS-US unpaired groups.

(immediate), $15 \mathrm{~min}, 1 \mathrm{hr}$, and $3 \mathrm{hr}$ after the end of training. There was a decrease in the level of performance with increasing retention intervals (Fig. 6). In addition, there was a significant overall effect of the training procedure administered. However, there was no significant interaction between training procedure and retention interval, meaning that, after different training procedures, the performance did not decline at significantly different rates. When the data from each retention interval (3 min, $15 \mathrm{~min}$, $1 \mathrm{hr}$, and $3 \mathrm{hr}$ ) were analyzed separately, there was a significant effect of the training procedure only at the $3 \mathrm{~min}$ retention test. Thus, the advantage conveyed by training with two trials at a 15 min ITI is lost by 15 min after training. It is noteworthy that, despite the low level of performance (performance indices of between 30 and 38 in most experiments) immediately after a single discrete training trial, there is still significant retention $3 \mathrm{hr}$ later. This indicates that, although the procedure produces modest conditioning, it is quite stable. 


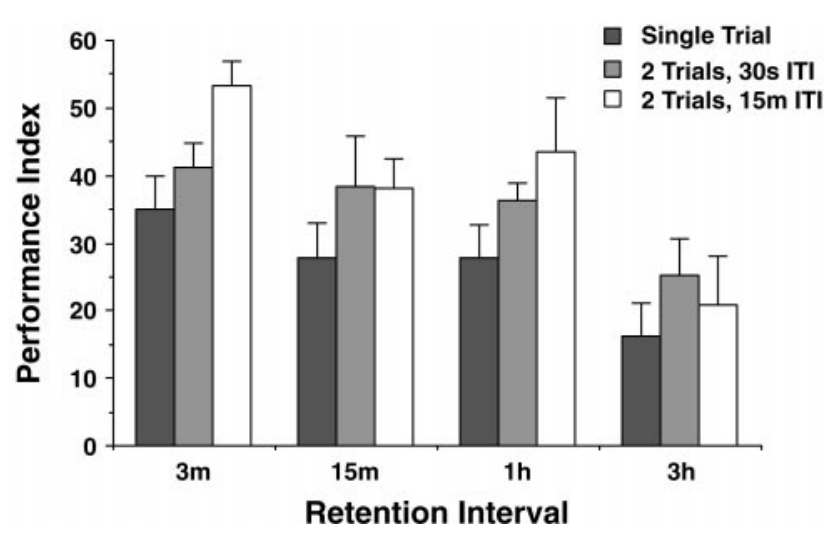

Figure 6. Memory after training. Performance evaluated at 3 min, 15 min, $1 \mathrm{hr}$, and $3 \mathrm{hr}$ after single trial, two trials massed, or two trials spaced conditioning. The spaced training produces higher performance immediately after training ( $3 \mathrm{~min}$ ), but the effect wanes rapidly, disappearing by $15 \mathrm{~min}$ after training. Statistics are as follows. There was a significant decrease in the level of performance with later retention intervals $\left(F_{(3,60)}=8.9 ; p<0.01 ; n=6\right.$ per training condition-retention interval). There was an overall significant effect of the training procedure $\left(F_{(2,60)}=\right.$ 5.3; $p<0.01)$, but no significant interaction between training procedure and retention interval $\left(F_{(6,60)}=0.5 ; \mathrm{NS}\right)$. Data analyzed from each retention interval showed a significant effect of spaced training only at 3 $\min \left(F_{(2,17)}=5.2 ; p<0.02 ; 15 \mathrm{~min}, F_{(2,17)}=1.0 ; \mathrm{NS} ; 1 \mathrm{hr}, F_{(2,17)}=1.9\right.$; $\mathrm{NS} ; 3 \mathrm{hr}, F_{(2,15)}=0.6$; NS).

\section{Phenotypes of memory mutants after short program training}

To investigate the utility of the short program in examining parameters of olfactory learning and memory, we tested several memory mutants along with controls in a simple battery consisting of one trial, two massed trials, and two spaced trials. This provided a rapid way of quantitating initial performance with very discrete CS and US presentations and for probing the normal enhancement of learning in spaced over massed training. We were interested in examining three different parameters of olfactory conditioning and comparing these with long program training. First, we wanted to determine whether we could observe differences between memory mutants using the short program. Second, some memory mutants have been rescued in long program conditioning using heat-inducible transgenes. We were interested in whether behavioral rescue would be revealed after short program conditioning. Third, many memory mutants of Drosophila are dominant for memory formation (Davis, 1996). We therefore were interested in whether dominance-recessivity relationships would be the same after short program conditioning.

The mutant rutabaga (rut) was initially isolated from genetic screens for defects in operant olfactory conditioning (Livingstone et al., 1984), and the gene was subsequently shown to encode an adenylyl cyclase (Levin et al., 1992). The allele used here, rut $^{2080}$, contains a P-factor at the locus and exhibits poor performance in long program olfactory classical conditioning (Han et al., 1992). Volado $(\mathrm{Vol})$ is a short-term memory mutant with a defective $\alpha$-integrin gene (Grotewiel et al., 1998). Vol $^{1}$, like $r u t^{2080}$, contains a P-factor at the locus and disrupts the expression of one of two RNAs (Vol-1, $4.6 \mathrm{~kb}$ ) from the locus, eliminating the $\alpha$-integrin gene product, VOL-L, encoded by this RNA (Grotewiel et al., 1998). A second allele, $\mathrm{Vol}^{2}$, contains a small deletion of the locus that selectively eliminates the alternative RNA transcript (Vol-s, $4.4 \mathrm{~kb}$ ) and the alternative $\alpha$-integrin isoform, VOL-S, encoded by this RNA (Grotewiel et al., 1998). Both of

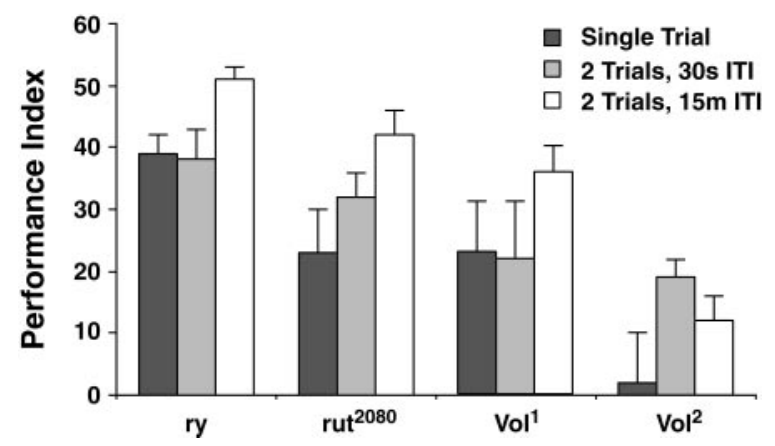

Figure 7. Performance of memory mutants after a single trial, two massed trials, and two spaced trials. Training of the $r y$ control produced spaced effects similar to those observed in Figures 3-5, with enhanced performance when given two spaced trials. All mutants performed poorly after any training condition with differences in how the three mutants respond to the different training conditions. The $r u t^{2080}$ mutant showed a significant effect from spaced training. $\mathrm{Vol}^{1}$ and $\mathrm{Vol}^{2}$ did not, although there was a trend in this direction for $\mathrm{Vol}^{1}$. Naive performance was noted with $\mathrm{Vol}^{2}$ given a single trial. Statistics are as follows. There was a significant effect of training and genotype (training, $F_{(2,60)}=3.3 ; p<0.05$; genotype, $\left.F_{(3,60)}=12.3 ; p<0.01 ; n=6\right)$. There was no significant interaction between training and strain $\left(F_{(6,60)}=1.5\right.$; NS), but inspection of the graph shows that there may be differences in how the strains reacted to the training procedures. Post hoc comparisons showed that the performance of the three mutants was lower overall than that of $r y$ and that the performance of $\mathrm{Vol}^{2}$ was lower than that of $\mathrm{Vol}^{1}$ or $r u t^{2080}$. When the data from each strain were analyzed separately, ry and $r u t^{2080}$ showed a significant effect of the training condition $\left(r y, F_{(2,15)}=4.0 ; p<.05 ; r_{t}{ }^{2080}\right.$, $\left.F_{(2,17)}=4.1 ; p<0.05\right)$, whereas $\operatorname{Vol}^{1}$ and $\operatorname{Vol}^{2} \operatorname{did} \operatorname{not}\left(\mathrm{Vol}^{1}, F_{(2,17)}=1.1\right.$; $\left.\mathrm{NS} ; \mathrm{Vol}^{2}, F_{(2,17)}=0.6 ; \mathrm{NS}\right)$.

these alleles show a dominant deficiency in 3 and 15 min memory after training in the long program for olfactory classical conditioning (Grotewiel et al., 1998). In addition, they both exhibit a performance decay that parallels that of the control at approximately the $50 \%$ level over a time course of $3 \mathrm{hr}$ after conditioning (Grotewiel et al., 1998). Behaviorally, therefore, they appear identical after long program conditioning. This led to the suggestion that the two $\alpha$-integrin isoforms encode redundant functions (Grotewiel et al., 1998). However, it is also possible that the failure to detect behavioral differences between the alleles is caused by an insensitivity of long program conditioning. Alternatively, the long program might obscure authentic differences.

Single-trial, short program training of $\mathrm{rut}^{2080}, \mathrm{Vol}^{1}$, and $\mathrm{Vol}^{2}$ showed that all mutants performed poorly immediately after conditioning (Fig. 7). In addition, they exhibited behavioral differences. The rut ${ }^{2080}$ and $\mathrm{Vol}^{1}$ mutants were sensitive to two-trial, spaced conditioning over two-trial, massed conditioning (see also Fig. 9). In this respect, both mutants were qualitatively like ry control animals. This phenotype is consistent with the interpretation that the mutants are defective in memory formation, or acquisition, a conclusion made for $\mathrm{Vol}^{1}$ from several other behavioral tests (C. Beck and R. Davis, unpublished observations). The $\mathrm{Vol}^{2}$ mutant performs strikingly different. Single-trial training failed to induce any conditioning whatsoever! Therefore, all three memory mutants show defective performance after short program training. In addition, the assay detected differences between $\mathrm{Vol}^{1}$ and $\mathrm{Vol}^{2}$ that were not detected by long program conditioning.

We also examined whether short program training with massed and spaced variations would be sensitive to behavioral rescue experiments using transgenes expressed from the heat shock (HS) promoter. Grotewiel et al. (1998) previously demonstrated that 


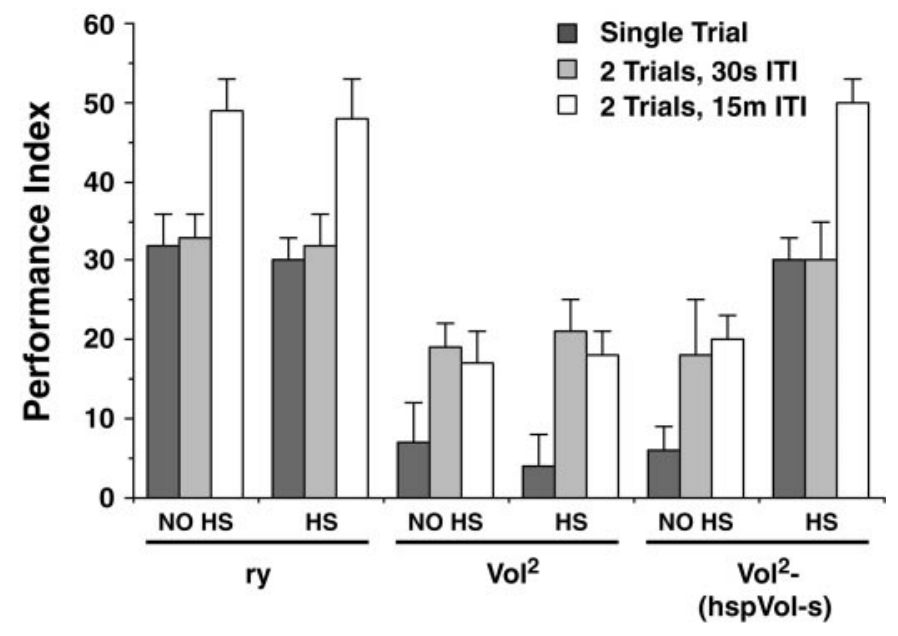

Figure 8. Behavioral rescue of spaced training performance. There was no effect of a $15 \mathrm{~min}$ HS on the performance of $r y$ flies after training with a single trial, two massed trials, and two spaced trials. $\mathrm{Vol}^{2}$ flies perform poorly after training; HS had no effect on performance. $\mathrm{Vol}^{2}(h s p V o l-s)$ flies performed like $\mathrm{Vol}^{2}$ mutants in the absence of HS but performed like ry controls after HS. Statistics are as follows. Three-factor ANOVA revealed an effect of genotype $\left(F_{(2,90)}=53.3 ; p<0.01\right)$, training procedure $\left(F_{(2,90)}=\right.$ 24.6; $p<0.01)$, and HS condition $\left(F_{(1,90)}=13.7 ; p<0.01\right) ; n=6$ for all groups. There was a significant interaction of genotype and training procedure $\left(F_{(4,90)}=3.4 ; p<0.05\right)$ and genotype and HS condition $\left(F_{(2,90)}=\right.$ $17.5 ; p<0.01)$. Fisher post hoc revealed that the single-trial groups and the two massed trial groups were not different from one another but were both significantly different from the two spaced trial groups for $r y$ (no HS), ry (HS), and $\mathrm{Vol}^{2}(h s p V o l-s)(\mathrm{HS})$. The single-trial groups were different from the two-trial massed and two-trial spaced groups for $\mathrm{Vol}^{2}$ (no HS) and $\mathrm{Vol}^{2}$ (HS). For the genotypes and training conditions $\left[\mathrm{Vol}^{2}\right.$ no HS, $\mathrm{Vol}^{2} \mathrm{HS}$, and $\mathrm{Vol}^{2}(\mathrm{hspVol}-2)$ no HS], one-sample, one-tailed $t$ tests (population mean of 0 ) showed significant performance only for the $\mathrm{Vol}^{2}(h \mathrm{spVol}-2)$ no HS group $\left.t_{(5)}=2.068 ; p<0.05\right)$. Neither the $\mathrm{Vol}^{2}$ no $\mathrm{HS}$ nor $\mathrm{Vol}^{2} \mathrm{HS}$ groups demonstrated any significant learning.

the performance deficit of $\mathrm{Vol}^{2}$ after long program conditioning could be rescued to control levels by induced expression of the Vol-s RNA, if HS was provided $3 \mathrm{hr}$ before training. Control ry flies, $\mathrm{Vol}^{2}$ mutants, and $\mathrm{Vol}^{2}$ mutants carrying the transgene $\left(\mathrm{Vol}^{2}\right.$, hspVol-s) were given HS or no HS $3 \mathrm{hr}$ before single-trial training, two massed trials, or two spaced trials. Immediate performance after training was measured.

As before, spaced training led to enhanced performance of $r y$ controls, which was independent of any HS treatment (Fig. 8). Similarly, $\mathrm{Vol}^{2}$ mutants (HS or no HS) and transgenic animals $\left(\mathrm{Vol}^{2}\right.$, hspVol-s; no HS) performed at naive levels after single-trial training and showed only modest performance after two massed or two spaced training trials. Most importantly, HS of $\mathrm{Vol}^{2}$, hspVol-s flies rescued the $\mathrm{Vol}^{2}$ behavioral phenotype to wild type qualitatively and quantitatively. Single-trial performance after HS was indistinguishable from controls, as was the failure of two massed trials to build significantly on single-trial performance. Two spaced trials given after HS produced performance equivalent to the control animals. This leads to two important conclusions. First, the short program can be used to evaluate behavioral rescue with conditional transgenes. Second, the $\mathrm{Vol}^{2}$ mutant was rescued by the conditional transgene not only after long program conditioning (Grotewiel et al., 1998) but also after short program training. Thus, the transgene appears to provide all essential functions for rescue in both behavioral assays.

The short program also proved to be a valuable assay for probing the dominance and recessivity of memory mutants. Pre-

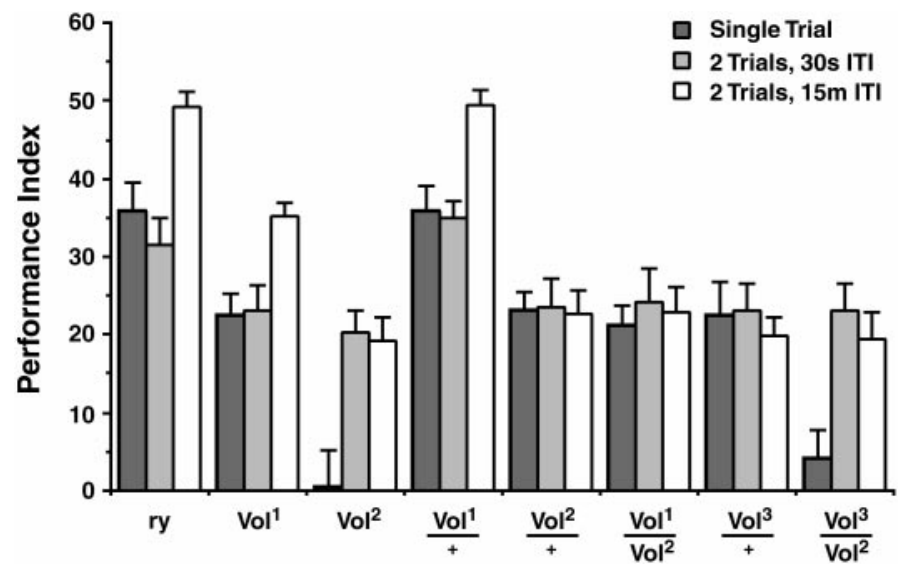

Figure 9. Dominance-recessivity after short program training. Flies of the genotypes listed were trained with a single trial, two massed trials, and two spaced trials. Immediate performance after training was measured. $\mathrm{Vol}^{1}$ and $\mathrm{Vol}^{2}$ both exhibited deficits as before. $\mathrm{Vol}^{1}$ was completely recessive after this training, because the performance of $\mathrm{Vol}^{1} /+$ was identical to the control. $\mathrm{Vol}^{2}$ was partially dominant for single-trial training, because $\mathrm{Vol}^{2} /+$ performance after this training was elevated over $\mathrm{Vol}^{2}$ homozygotes but was not at wild-type levels. The $\mathrm{Vol}^{2}$ phenotype after two massed trials or two spaced trials, however, was completely dominant, because the performance of $\mathrm{Vol}^{2} /+$ with this training was identical to $\mathrm{Vol}^{2}$ homozygotes. The partially dominant phenotype (single trial) and completely dominant phenotype (two trials, massed or spaced) of $\mathrm{Vol}^{3}$ observed in $\mathrm{Vol}^{3} /+$ flies is accounted for by the loss of the Vol-s transcript, given the behavioral similarities with $\mathrm{Vol}^{2} /+$ and $\mathrm{Vol}^{1} / \mathrm{Vol}^{2}$ flies. Conditioning after a single trial is completely dependent on the presence of at least one copy of the Vol-s transcription unit (behavior of $\mathrm{Vol}^{3} / \mathrm{Vol}^{2}$ and $\mathrm{Vol}^{2}$ homozygotes). Statistics are as follows: two-factor ANOVA revealed an effect of genotype $\left(F_{(7,120)}=28.0 ; p<0.01\right)$, training procedure $\left(F_{(2,120)}=16.2 ; p<0.01\right)$, and a significant interaction $\left(F_{(14,120)}=3.7 ; p<0.01\right) ; n=6$ for all groups. Fisher post hoc revealed that the single-trial groups and the two massed trial groups are not different from one another but are both significantly different from the two spaced trial groups for $r y, \mathrm{Vol}^{1}$, and $\mathrm{Vol}^{1} /+$. The single-trial groups were different from the two-trial massed and two-trial spaced groups for $\mathrm{Vol}^{2}$ and $\mathrm{Vol}^{3} / \mathrm{Vol}^{2}$.

vious research established that the $\mathrm{Vol}^{1}$ and $\mathrm{Vol}^{2}$ alleles are both dominant for performance after long program training when tested at 3 and 15 min after conditioning (Grotewiel et al., 1998). We retested these alleles, along with a new allele, Vol $^{3}$, a 925 bp deletion that simultaneously removes both the Vol-1 and Vol-s transcription units (J. Rohrbough, M. S. Grotewiel, R. L. Davis, and K. Broadie, unpublished observations). These alleles offered the opportunity to eliminate one copy of the Vol-1 transcription unit $\left(\mathrm{Vol}^{1} /+\right)$, one copy of the Vol-s transcription unit $\left(\mathrm{Vol}^{2} /+\right)$, both copies of Vol-1 $\left(\mathrm{Vol}^{1}\right)$, both copies of Vol-s $\left(\mathrm{Vol}^{2}\right)$, one copy each of Vol-1 and Vol-s $\left(\mathrm{Vol}^{3} /+\right.$ and $\left.\mathrm{Vol}^{1} / \mathrm{Vol}^{2}\right)$, and both copies of Vol-s along with one copy of Vol-l $\left(\mathrm{Vol}^{3} / \mathrm{Vol}^{2}\right)$. The different genotypes were trained with a single trial, two massed trials, or two spaced trials (Fig. 9).

In contrast to long program conditioning, the $\mathrm{Vol}^{1}$ allele was completely recessive after short program conditioning (Fig. 9). This indicated that a single copy of the Vol-1 transcription unit is sufficient for normal behavior in this assay. Furthermore, the behavior of $\mathrm{Vol}^{1}$ homozygotes was reduced overall but appeared qualitatively like the control by showing the normal enhancement because of spaced training. These data suggested that the VOL-L integrin is not an essential factor for conditioning but modulates the effectiveness of the conditioning.

The $\mathrm{Vol}^{2}$ allele was partially dominant for performance after 
single-trial and two-trial massed conditioning ( $\mathrm{Vol}^{2} /+$ performance). However, at least one copy of the Vol-s transcription unit was required for any conditioning to occur after a single trial, because genotypes that remove both copies of the Vol-s transcription unit $\left(\mathrm{Vol}^{2}\right.$ and $\mathrm{Vol}^{3 /} \mathrm{Vol}^{2}$ ) exhibited absolutely no conditioning whatsoever! Thus, the VOL-S integrin is an essential factor for single-trial conditioning. Furthermore, $\mathrm{Vol}^{2}$ is completely dominant for the memory enhancement that comes with spaced training, because no effect of spaced training was observed in genotypes that remove one or both copies of the Vol-s transcription unit $\left(\mathrm{Vol}^{2}, \mathrm{Vol}^{2} /+, \mathrm{Vol}^{1} / \mathrm{Vol}^{2}, \mathrm{Vol}^{3} /+\right.$, and $\left.\mathrm{Vol}^{3} / \mathrm{Vol}^{2}\right)$. The $\mathrm{Vol}^{2}$ allele, therefore, has a stronger effect overall on conditioning than the $\mathrm{Vol}^{1}$ allele. These behavioral genetic data suggest that the two integrin isoforms play qualitatively different roles in the processes underlying memory formation, a conclusion suggested by other behavioral (Beck and Davis, unpublished observations) and physiological (Rohrbough, Grotewiel, Davis, and Broadie, unpublished observations) studies.

\section{Electric shock, stress, and odor salience}

One complication of long program training is that the rapid shock pulses (12, with 1 per $5 \mathrm{sec}$ ) alters the salience of the odor cues used for training, as assayed by the normal avoidance of the aversive odor cues (Preat, 1998). This effect has been ascribed to stress produced by the shock pulses. In addition, some learning mutants respond more acutely than controls to this stressful situation, producing the worrisome situation that odor cues during testing may be more salient to controls than to certain memory mutants (Preat, 1998).

We assayed the effect of shock pulses delivered in mock training trials of the short program for a single trial, two massed trials, or two spaced trials. Electric shock was delivered normally in the mock training, but odor cues were replaced by fresh air. Afterwards, the odor avoidance was measured and compared with animals receiving no pretreatment. The $r y$ control and the $r u t^{2080}$ mutant avoided the two odors used for training to the same extent when given a choice of fresh air in a T-maze (Fig. 10). Electric shock pulses delivered according to the time schedule for training with a single trial, two massed trials, or two spaced trials did not alter subsequent avoidance of the odors. Similar results have been obtained for $\mathrm{Vol}^{1}$ and $\mathrm{Vol}^{2}$ (Beck and Davis, unpublished observations). Thus, short program training eliminated the confounding factor of stress and odor desensitization that occurs with long program olfactory classical conditioning.

\section{DISCUSSION}

Insects have provided considerable insights into the molecular and cellular mechanisms underlying learning and memory (Hammer and Menzel, 1995; Davis, 1996). Drosophila has provided for a behavioral genetic approach to learning; mutants have been selected, the genes have been cloned, gene expression patterns have been elucidated, and models have been built to explain the role of these molecules and cells in learning. This molecular genetic approach, however, requires the existence of robust and dependable behavioral paradigms that can reliably measure various aspects of memory formation and stability to help understand how different mutants, and therefore individual molecules, participate in these processes. Although Drosophila has been shown to be capable of learning through many different sensory modalities, olfactory learning has been the most intensely studied type of learning using the long program as the standard assay.

We have developed an alternative assay that offers numerous

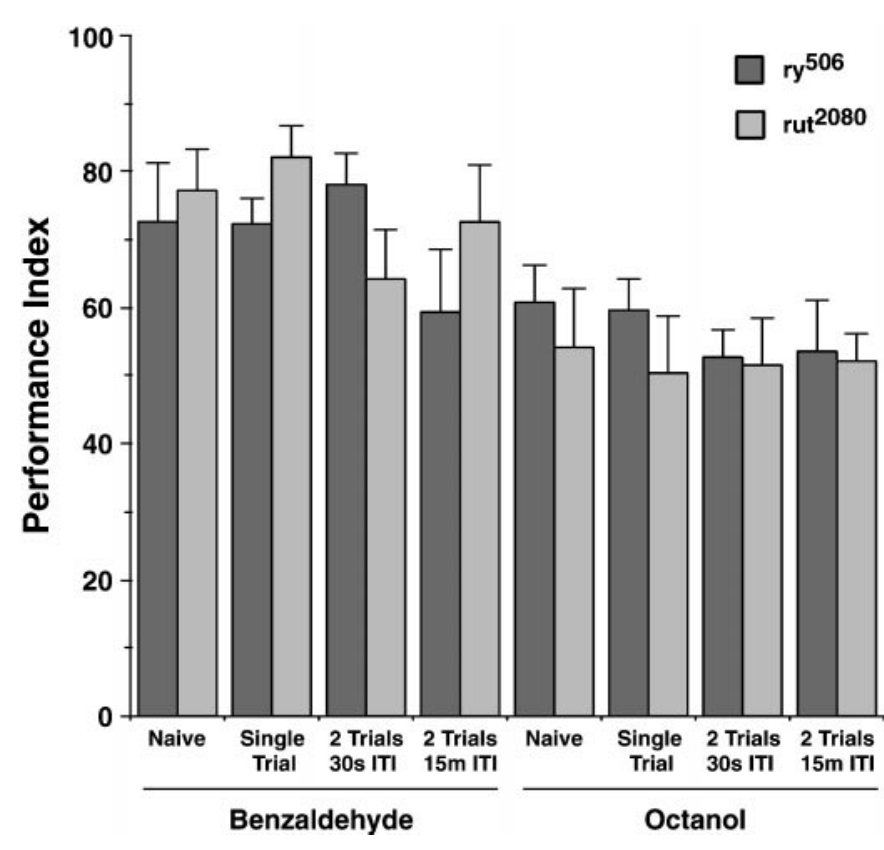

Figure 10. Odor avoidance after mock conditioning. Odor avoidance to benzaldehyde or octanol was measured after mock training of ry and $r u t^{2080}$. Statistics are as follows. Two-factor ANOVA revealed no effect of genotype $\left(F_{(1,56)}=0.49 ; \mathrm{NS}\right)$ or training procedure $\left(F_{(3,56)}=1.05 ; \mathrm{NS}\right)$ using benzaldehyde as the odor. Two-factor ANOVA showed no effect of genotype $\left(F_{(1,56)}=0.94 ; \mathrm{NS}\right)$ or training procedure $\left(F_{(3,56)}=0.28 ; \mathrm{NS}\right)$ using octanol as the odor; $n=8$ for all groups.

advantages. First, it is reliable and highly reproducible. The figures included here present data from 17 independent assays (each with $n \geq 6$ ) in which $r y$ controls were trained with a single trial. The performance indices for these 17 experiments range from 28 to 39, with a mean of 33.3. The figures show data from 13 and 14 independent experiments, respectively, in which the controls were trained with two massed trials or two spaced trials. The range observed for two massed trials was 31-41 with a mean of 33.8; the range observed for two spaced trials was $48-53$, with a mean of 51. These data illustrate that the short program generates highly reproducible behavior. Second, the discrete stimuli used by the program provide a method for dissecting the rate of memory formation, or acquisition. Multiple trials presented sequentially can be used to compare the memory formed in controls and mutants after one, two, or three trials, for example, to obtain measurements of the rate of memory formation. This is impossible with the long program because the training protocol induces ceiling levels of performance after a single trial. Third, memory stability can be measured immediately after single-trial training to survey the state of very early memory and its subsequent decay. Fourth, the unique behavioral enhancement after two spaced trials over single-trial conditioning or two massed trials offers another criterion by which to compare mutants and controls. As shown here, certain mutants $\left(\mathrm{Vol}^{1}\right.$ and $\left.\mathrm{Vol}^{2}\right)$ differ dramatically in their responses to this training. This may provide insights into the mechanisms that operate to produce the enhancement, as well as help to dissect the roles of various gene products in memory formation. Finally, the short program circumvents several problems associated with long program training. The spacing of shock pulses during long program training may produce some extinction during periods when the CS is present but the US is not. The intense shock pulses are thought to produce a stressful situation 
that alters the salience of odors. The salience change may be different from strain to strain, yielding a situation that may produce misinterpretations about their relative behavior (Preat, 1998). Nevertheless, long program conditioning will not be replaced by the short program procedure, because the examination of longer-term memory requires the induction of high levels of initial performance. We believe that experiments using both schedules may yield the most meaningful information about each mutant.

The enhancement in performance with two spaced trials and seven spaced trials is intriguing. Some previous studies have examined the effects of spaced training on the duration of memory (Carew et al., 1972; Tully et al., 1994; Kogan et al., 1997). This enhancement, in contrast, is on immediate performance after the training. Furthermore, in contrast to the effects of inhibitors of protein synthesis on memory duration after spaced training (Tully et al., 1994), the effect observed here was independent of cycloheximide treatment. Thus, we conclude that the enhancement in performance early after training as a result of spaced training is mechanistically distinct from the long-term and protein synthesis-dependent memory that spaced training can also induce. It seems likely that the early effect may be mediated by changes in second messenger metabolism and intracellular signaling pathways rather than by alterations in gene expression and protein synthesis.

Finally, the use of the short program has yielded new information about the memory mutant Volado. Although flies carrying the alleles $\mathrm{Vol}^{1}$ or $\mathrm{Vol}^{2}$ appear quite similar in memory formation and stability after long program training, they are very distinct after short program training. The dominance-recessivity relationships for various behavioral parameters are different between the two mutants. These relationships are not easy to interpret at this time, although thresholds in the requirement for VOL-L and VOL-S or different molecular functions for the two integrins offer possible explanations. The fact that VOL-L and VOL-S differ at their $\mathrm{N}$ termini (a region involved in ligand binding) lead us to believe that they have different functions.

\section{REFERENCES}

Byers D, Davis RL, Kiger Jr JA (1981) Defect in cyclic AMP phosphodiesterase due to the dunce mutation of learning in Drosophila melanogaster. Nature 289:79-81.

Carew TJ (1996) Molecular enhancement of memory formation. Neuron 16:5-8.

Carew TJ, Pinsker HM, Kandel ER (1972) Long-term habituation of a defensive withdrawal reflex in Aplysia. Science 175:451-454.

Chen CN, Denome S, Davis RL (1986) Molecular analysis of cDNA clones and the corresponding genomic coding region of the Drosophila dunce + locus, the structure gene for cAMP phosphodiesterase. Proc Nat Acad Sci USA 86:3599-3603.

Clark RE, Squire LR (1998) Classical conditioning and brain systems: the role of awareness. Science 280:77-81.

Davis RL (1993) Mushroom bodies and Drosophila learning. Neuron 11:197-208.

Davis RL (1996) Physiology and biochemistry of Drosophila learning mutants. Physiol Rev 76:299-317.

Davis RL, Kiger Jr JA (1981) . Dunce mutants of Drosophila melanogaster: mutants defective in the cyclic AMP phosphodiesterase enzyme system. J Cell Biol 90:101-107.

DeBelle JS, Heisenberg M (1994) Associative odor learning in Drosophila abolished by chemical ablation of mushroom bodies. Science 263:692-695.

Dudai Y, Jan Y-N, Byers D, Quinn W, Benzer S (1976) Dunce, a mutant of Drosophila deficient in learning. Proc Natl Acad Sci USA 73:1684-1688.

Fanselow MS, Tighe TJ (1988) Contextual conditioning with massed versus distributed unconditional stimuli in the absence of explicit conditional stimuli. J Exp Psychol 14:187-199.

Goldstone RL (1998) Perceptual learning. Annu Rev Psychol 49:585-612.

Griffith LC, Verselis LM, Aitken KM, Kyriacou CP, Danho W, Greenspan RJ (1993) Inhibition of calcium/calmodulin-dependent protein kinase in Drosophila disrupts behavioral plasticity. Neuron 10:501-509.

Grotewiel MS, Beck CDO, Wu K-H, Zhu X-R, Davis RL (1998) Integrin-mediated short-term memory in Drosophila. Nature 391:455-460.

Hammer M, Menzel R (1995) Learning and memory in the honeybee. J Neurosci 15:1617-1630.

Han P-L, Levin LR, Reed RR, Davis RL (1992) Preferential expression of the Drosophila rutabaga gene in mushroom bodies, neural centers for learning in insects. Neuron 9:619-627.

Hermitte G, Pedreira ME, Tomsic D, Maldonado H (1999) Context shift and protein synthesis inhibition disrupt long-term habituation after spaced, but not massed, training in the crab Chasmagnathus. Neurobiol Learn Mem 71:34-49.

Jellies JA (1981) Associative olfactory conditioning in Drosophila melanogaster and memory retention through metamorphosis. Master thesis, Illinois State University.

Kogan JH, Frankland PW, Blendy JA, Coblentz J, Marowitz Z, Silva AJ (1997) Spaced training induces normal long-term memory in CREB mutant mice. Curr Biol 7:1-11.

Levin LR, Han P-L, Hwang PM, Feinstein PG, Davis RL, Reed RR (1992) The Drosophila learning and memory gene rutabaga encodes a $\mathrm{Ca}^{2+} /$ calmodulin-responsive adenylyl cyclase. Cell 68:479-489.

Livingstone MS, Sziber PP, Quinn WG (1984) Loss of calcium/calmodulin responsiveness in adenylate cyclase of rutabaga, a Drosophila learning mutant. Cell 137:205-215.

Lubow RE (1997) Latent inhibition as a measure of learned inattention: some problems and solutions. Behav Brain Res 88:75-83.

Martin KC, Casadio A, Zhu YE, Rose JC, Chen M, Bailey CH, Kandel ER (1997) Synapse-specific, long-term facilitation of Aplysia sensory to motor synapses: a function for local protein synthesis in memory storage. Cell 91:927-938.

Miller EK (1999) The prefrontal cortex: complex neural properties for complex behavior. Neuron 22:15-17.

Montarolo PG, Goelet P, Castellucci VF, Morgan J, Kandel ER, Schacher S (1986) A critical period for macromolecular synthesis in long-term heterosynaptic facilitation in Aplysia. Science 234:1249-1254.

Muzzio IA, Ramirez RR, Talk AC, Matzel LD (1999) Interactive contributions of intracellular calcium and protein phosphatases to massedtrials learning deficits in Hermissenda. Behav Neurosci 113:103-117.

Nighorn A, Healy MJ, Davis RL (1991) The cyclic AMP phosphodiesterase encoded by the Drosophila dunce gene is concentrated in the mushroom body neuropil. Neuron 6:455-467.

Preat T (1998) Decreased odor avoidance after electric shock in Drosophila mutants biases learning and memory test. J Neurosci 18:8534-8538.

Rescorla RA (1988) Behavioral studies of Pavlovian conditioning. Annu Rev Neurosci 11:329-352.

Skoulakis EMC, Davis RL (1996) Olfactory learning deficits in mutants for Leonardo, a Drosophila gene encoding a 14-3-3 protein. Neuron 17:931-944.

Skoulakis EMC, Kalderon D, Davis RL (1993) Preferential expression of the catalytic subunit of PKA in the mushroom bodies and its role in learning and memory. Neuron 11:197-208.

Spieler DH, Balota DA (1996) Characteristics of associative learning in younger and older adults: evidence from an episodic priming paradigm. Psychol Aging 11:607-620.

Tully T, Quinn WG (1985) Classical conditioning and retention in normal and mutant Drosophila melanogaster. J Comp Physiol 157:263-277.

Tully T, Preat T, Boynton SC, DelVecchio M (1994) Genetic dissection of consolidated memory in Drosophila. Cell 79:35-47.

Weiner I (1990) Neural substrates of latent inhibition: the switching model. Psychol Bull 108:442-461.

Wolf R, Wittig T, Lui L, Wustmann G, Eyding D, Heisenberg M (1998) Drosophila mushroom bodies are dispensable for visual, tactile, and motor learning. Learn Mem 5:166-178.

Yin H, Barnet RC, Miller RR (1994) Trial spacing and trial distribution effects in Pavlovian conditioning: contributions of a comparator mechanism. J Exp Psychol 20:123-134. 